\title{
KM3NeT/ARCA Event Reconstruction Algorithms
}

\section{Karel Melis*}

Nikhef Institute for Subatomic Physics, Amsterdam, The Netherlands

E-mail: kmelis@nikhef.nl

\section{Aart Heijboer}

Nikhef Institute for Subatomic Physics, Amsterdam, The Netherlands

E-mail: aart.heijboer@nikhef.nl

\section{Maarten de Jong}

Nikhef Institute for Subatomic Physics, Amsterdam, The Netherlands

E-mail: mjg@nikhef.nl

\section{On behalf of the KM3NeT Collaboration}

By using clear water as detection medium, the KM3NeT Neutrino detector currently under construction in the Mediterranean Sea will be able to study the high-energy neutrino sky with an unprecedented resolving power for each neutrino flavour. Simulations and validation in the ANTARES detector, show that degree-level resolution for cascade events will be achieved, opening up the possibility to do neutrino astronomy with these neutrinos. For muon neutrinos, the resolution will surpass 0.1 degree at high energies, offering an order of magnitude more resolving power compared to the current state of the art. We will discuss features of the water that make this possible, as well as the latest reconstruction methods for tracks and cascades.

35th International Cosmic Ray Conference - ICRC2017

10-20 July, 2017

Bexco, Busan, Korea

\footnotetext{
* Speaker.
} 


\section{Introduction}

The KM3NeT Collaboration aims to 1) determine the neutrino mass hierarchy using the KM3NeT/ORCA detector and 2) identify and study the sources of high-energy neutrinos using the KM3NeT/ARCA detector. The infrastructure will consist of three-dimensional grids of photomultiplier tubes (PMTs) at three locations on the Mediterranean sea-bed to detect the Čherenkov light emitted by the secondaries produced in neutrino interactions. Depending on the interaction type and neutrino flavour, three event classes can be distinguished: track-like events from chargedcurrent (CC) muon-neutrino events, shower-like events from neutral-current (NC) interactions or $\mathrm{CC}$ electron-neutrino interactions and $\mathrm{CC}$ tau-neutrino interactions leading to so-called doublebang events. The unprecedented resolutions achieved with the algorithms used to determine the neutrino properties of high-energy track-like and shower-like events in the KM3NeT/ARCA detector will be highlighted in this contribution.

\section{The KM3NeT/ARCA Detector}

In both the ORCA and ARCA detector of KM3NeT, 31 PMTs with accompanying dataprocessing and calibration devices are housed in a pressure-resistant glass sphere called of the Digital Optical Module (DOM) (figure 1). DOMs are installed in Detection Units (DUs); vertical lines with 18 DOMs each, kept vertical by a buoy system; 115 DUs form a detector building block.

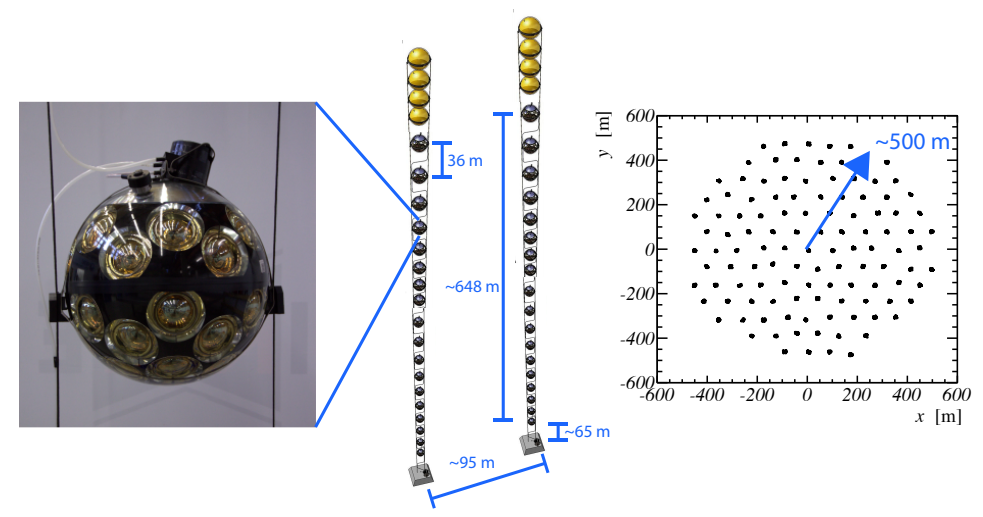

Figure 1: Schematic outline of a KM3NeT/ARCA building block.

The vertical and horizontal DOM spacing is optimized for the main physics objective of the detector [1] [2]. For KM3NeT/ORCA, one densely spaced building block is foreseen near Toulon, France. For KM3NeT/ARCA, two building blocks are planned offshore Sicily, Italy. Since December 2015 and May 2016 respectively, two detection units of the ARCA detector have been successfully taking data [3] [4].

For each PMT, the arrival time and the time over threshold of the analogue pulses produced by detected photons are measured and sent to shore for event triggering and further analysis using a network of optical fibers. This network also provides the necessary electrical power to the DOMs. The on-shore data-acquisition and event triggering software writes all hits within a time window of approximately $\pm 10 \mu \mathrm{s}$ when a cluster of coincident hits compatible with a track or shower hypothesis is detected, thus providing all necessary information for the event reconstruction algorithms. 


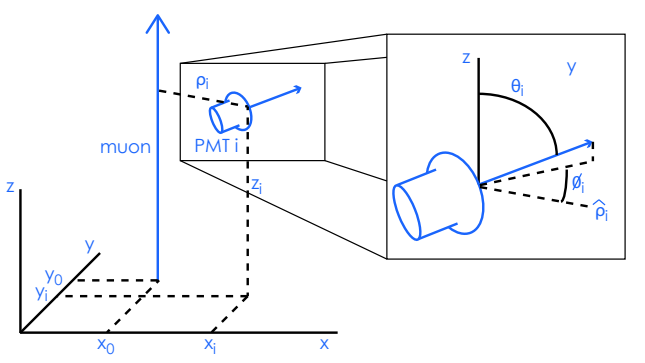

Figure 2: Coordinate system used in the muon trajectory fit.

\section{Monte Carlo Simulations}

The presented results are obtained using Monte Carlo simulated events in a single ARCA building block using the KM3NeT software packages genhen, $\mathrm{km} 3$ and Jpp for the simulation of the secondary particles produced in neutrino interactions, photon propagation through the water and detector response, respectively [5]. If not mentioned explicitly, a neutrino energy spectrum of $E^{-2.46}$ is assumed.

\section{Muon Track Reconstruction}

The objective of the event reconstruction is the determination of the trajectory and energy of ultra-relativistic muons. The muon trajectory can be described by five independent parameters: its direction and the time and position along the trajectory. The main difficulty in the reconstruction of the muon trajectory is the non-linearity of the problem. This is overcome by the use of a prefit, providing a suitable set of start values for the final trajectory fit.

Prefit Given an assumed muon direction, a coordinate system can be defined in which the muon travels along the z-axis, crossing the $z=0$ plane at coordinates $x_{0}, y_{0}$ at time $t_{0}$ (figure 2). In the absence of scattering and dispersion of light, the expected arrival time of the Čerenkov photons $\hat{t}_{i}$ on a PMT $i$ can then be expressed as:

$$
\begin{aligned}
\hat{t}_{i} & =t_{0}+\frac{z_{i}}{c}+\tan \left(\theta_{c}\right) \frac{\rho_{i}}{c} \\
\rho_{i} & =\sqrt{\left(x_{i}-x_{0}\right)^{2}+\left(y_{i}-y_{0}\right)^{2}}
\end{aligned}
$$

where $\rho_{i}$ is the minimum distance of approach of the muon to the PMT, $z_{i}$ is the distance from the PMT to the $z=0$ plane, $c$ is the speed of light in vacuum and $\theta_{c}$ the characteristic Čerenkov angle. By considering all pairs of consecutive hits $i, j$, the problem can be formulated as a readily-solvable matrix equation holding the following relations: 


$$
\begin{aligned}
t_{j}^{\prime 2}-t_{i}^{\prime 2}-2\left(t_{j}^{\prime}-t_{i}^{\prime}\right) t_{0}= & x_{j}^{2}-x_{i}^{2}-2\left(x_{j}-x_{i}\right) x_{0} \\
& +y_{j}^{2}-y_{i}^{2}-2\left(y_{j}-y_{i}\right) y_{0} \\
t_{i}^{\prime}= & t_{i} \cdot c / \tan \left(\theta_{c}\right)-z_{i} / \tan \left(\theta_{c}\right)
\end{aligned}
$$

This linear fit is sensitive to outliers from optical background hits -caused by ${ }^{40} \mathrm{~K}$ decays and bioluminescence- and strongly scattered photons. In order to exclude these, a cluster of causally related hits is selected from the data. For a limited number of causally related hits, i.e. $n \leq N$, the linear fit is applied to all permutations of $N, N-1, \ldots, m$ hits, where $m$ is some minimal number of hits (typically $m=4$ ) and $N=8$. The solution with the largest number of hits is selected, unless a solution exists which yields a $\chi^{2}$ smaller by (typically) three standard deviations or more when one hit less is used. For events with more than eight hits, i.e. $n>N$, the fit is applied to all hits. Possible outliers are subsequently removed as long as their contribution to the total $\chi^{2}$ is larger than (typically) three standard deviations.

The procedure described above is repeated for a scan of assumed track directions with a typical grid angle of one degree. A set of 12 best fitted test directions provides at least one suitable start value for the subsequent fit stage in $90 \%$ of the events, where the fit quality $Q$ is quantified as:

$$
Q=\mathrm{NDF}-0.25 \times\left(\chi^{2} / \mathrm{NDF}\right)
$$

where NDF refers to the number of degrees of freedom in the fit.

Muon Trajectory Fit Starting from the twelve best fitted directions in the prefit, a maximum likelihood search is performed using the Levenberg-Marquardt method in which all five independent trajectory parameters are fitted simultaneously. In the likelihood function, the PMT response is described by a set of probability density functions (PDFs):

$$
\mathscr{L}=\prod_{h i t P M T s}\left[\frac{\partial P}{\partial t}\left(\rho_{i}, \theta_{i}, \phi_{i}, \Delta t\right)\right]
$$

where $\rho_{i}$ is the minimum distance of approach of the muon to the PMT $i, \theta_{i}$ and $\phi_{i}$ describe the orientation of the PMT (see figure 2) and $\Delta t$ is the difference between the expected (equation 4.1) and measured arrival time of the light. The set of PDFs are calculated semi-analytically for unscattered and single-scattered light from Čherenkov radiation and from energy losses of the muon -such as from Bremsstrahlung- and include the effects of dispersion as well as the optical background rate and the quantum efficiency $(\mathrm{QE})$, angular acceptance and transit time spread of the PMTs [3]. To provide partial derivates and to limit computational time, the PDFs are evaluated using interpolating functions in 4D.

In figure 4, the muon arrival time PDF is shown for a PMT looking towards and another one facing away from a muon track of $1 \mathrm{TeV}$ at a distance of closest approach of 50 meters. As can be seen, the PDFs have a very sharp peak exceeding the expected background rate by many orders of magnitude. This sharply peaked PDF is the key performance ingredient of the track reconstruction, resulting in an unprecedented angular resolution better than 0.1 degrees at high energies (figure 5). For neutrino energies $\lesssim 10^{4} \mathrm{GeV}$, the error is dominated by the intrinsic scattering angle of the neutrino interaction. 


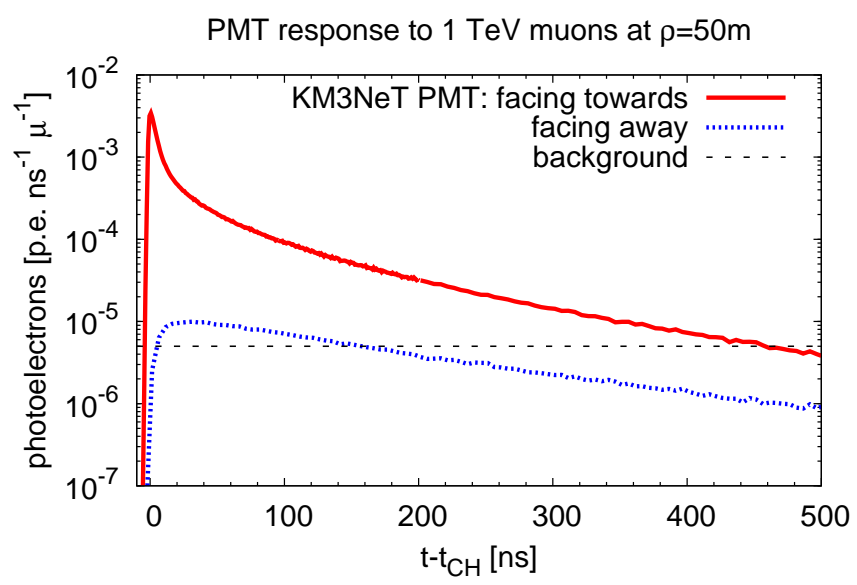

Figure 4: Expected number of photons hitting a PMT facing/facing away from a muon track of 1 $\mathrm{TeV}$ at a distance of closest approach $\rho_{i}$ of 50 meters. The expected number of background hits given a typical singles rate of $5 \mathrm{kHz}$ is given by the horizontal dotted line. Figure from [1].

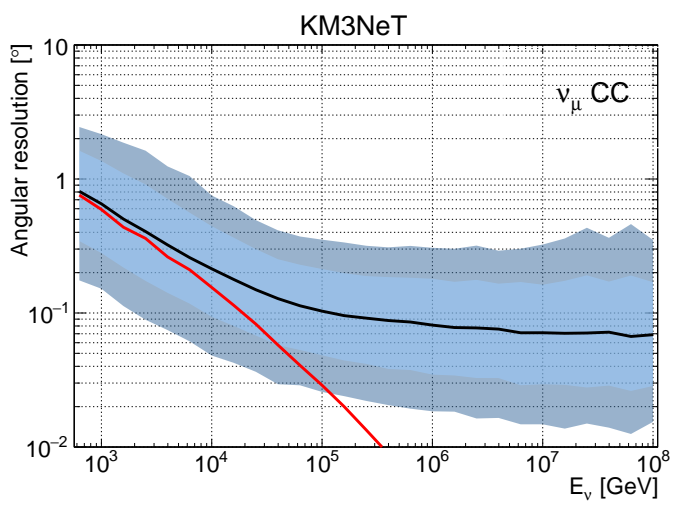

Figure 5: Median angular error (angle between reconstructed track and neutrino true direction) of the final muon trajectory fit as function of the neutrino true energy. The coloured bands represent the $68 \%$ and $90 \%$ quantiles. The red line gives the median angle between the true neutrino and muon directions.

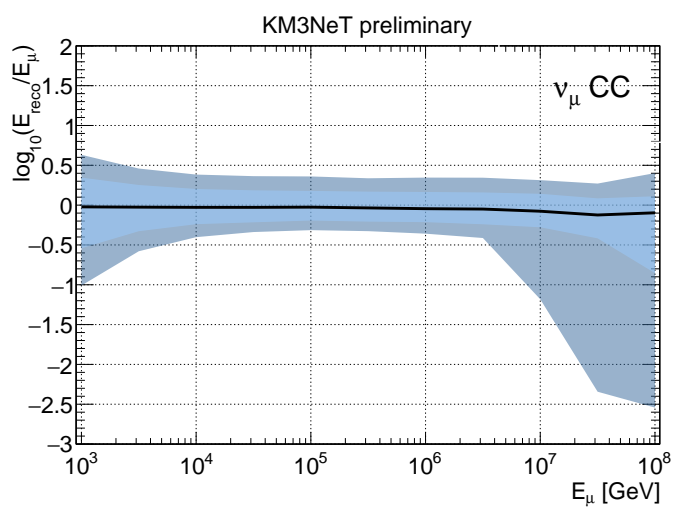

Figure 6: Median energy resolution (solid line) of the muon energy fit as function of the muon true energy with $68 \%$ and $90 \%$ quantiles. The standard deviation of the $\log _{10}\left(E_{\text {reco }} / E_{\mu}\right)$ distribution is less than 0.3 .

Muon Energy Fit With the muon trajectory determined, the energy of the muon is fitted using the spatial distribution of hit and non-hit PMTs. The probability of a PMT being hit is obtained from the same PDFs as discussed in the previous section. All PMTs within a predefined road width (200 meters by default) around the muon trajectory are used for which the calculated hit probability is compared to the occurrence of a hit. In figure 6, the fitted muon energy is compared to the true muon energy. A resolution better than 0.3 in $\log _{10}\left(E_{\text {reco. }} / E_{\mu}\right)$ is achieved. 


\section{Cascade Reconstruction}

It has been realized that the capability to reconstruct cascade type neutrino events will dramatically increase the science reach of large volume neutrino telescopes. This was illustrated further by the discovery by IceCube of cosmic neutrinos [6]. The default KM3NeT cascade reconstruction algorithm follows two stages; after a fit of the shower vertex based on the hit times, the shower direction and energy are estimated using the distribution of PMTs being hit or not.

Vertex fit The shower vertex fit uses a high-purity sample of hits by selecting coincidence hits on the same DOM within $20 \mathrm{~ns}$. In this sample, background hits from optical backgrounds from ${ }^{40} \mathrm{~K}$ decays and bioluminescence may still be present, but their influence on the fit is further suppressed by the use of an M-estimator score-function:

$$
\begin{aligned}
& M=\sum_{\text {hits }} \sqrt{1+\left(t_{i}-\hat{t}_{i}\right)^{2}} \\
& \hat{t}_{i}=t_{0}+\frac{n}{C} r_{i}
\end{aligned}
$$

in which the time of a hit $t_{i}$ on a DOM $i$ at distance $r_{i}$ to the shower position is compared with the expected hit time $\hat{t}_{i}$ of a spherical wavefront expanding with velocity $c / n$ from the shower position at time $t_{0}$. The best fit shower position and $t_{0}$ are found using root's TMath::Minimizer interface [7], starting from the center of gravity of the hits.

The performance of the vertex fit is presented in figure 7, which shows the distance between the neutrino interaction vertex and the reconstructed shower position, decomposed into components along (left) and perpendicular (right) to the shower axis (i.e. neutrino direction). All events are reconstructed and no quality cuts have been applied. The increase of shower elongation with energy is clearly visible in the longitudinal component. If the reconstructed vertex is compared with the point along the shower axis at which most light is emitted (the shower maximum), an accuracy of a few decimeters is achieved. This position resolution has paved the way for a tau double-bang event reconstruction algorithm currently under development.

Shower direction fit Using the fitted shower vertex as pivot point, the shower direction $\hat{d}_{S}$ and energy $E_{S}$ are found using a maximization procedure starting from 12 isotropic starting directions using TMath::Minimizer [7]. The (log)likelihood function used is purely based on hit/not hit information:

$$
\begin{aligned}
\log \mathscr{L} & =\sum_{\text {empties }} \log \left[P_{i}^{\text {nohit }}\right]+\sum_{\text {hitPMTs }} \log \left[1-P_{i}^{\text {nohit }}\right] \\
P_{i}^{\text {nohit }} & =\exp \left[-\mu_{\text {sig }}\left(r_{i}, z_{i}, a_{i}, E_{S}\right)-R_{b g} \cdot T\right]
\end{aligned}
$$

in which $\mu_{s i g}\left(r_{i}, z_{i}, a_{i}, E_{S}\right)$ give the expected number of photons on a PMT $i$ in the coordinate system outlined in figure 3. In order to minimize the influence of optical backgrounds, only hits within a time window $T$ ranging from $-100 \mathrm{~ns}$ to $+900 \mathrm{~ns}$ around the expected hit time from the vertex fit (equation 5.2) are considered. The expected number of hits from the shower $\mu_{\text {sig }}$ is evaluated using interpolation of a three-dimensional histogram depending on $r_{i}, z_{i}$ and $a_{i}$ at a shower energy of 1 $\mathrm{PeV}$ (figure 8). The expected number of hits at different shower energies is calculated using the fact that the number of emitted photons scales linearly with the shower energy $E_{S}$. 

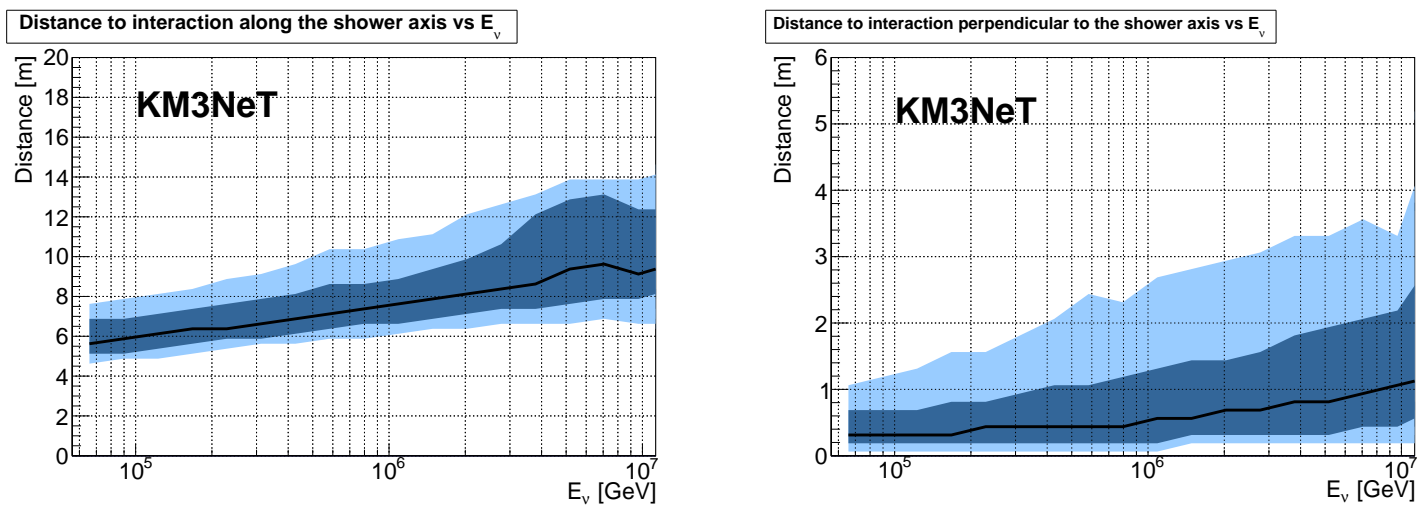

Figure 7: Performance of the shower vertex fit. Dark and light blue bands contain 68 and $90 \%$ of the distribution respectively; the black line is the median. The left plot shows the distance from the reconstructed position to the neutrino interaction point, projected on the shower axis. The right plot is the distance from the reconstructed position to the shower axis. All events are reconstructed and no quality cuts have been applied.
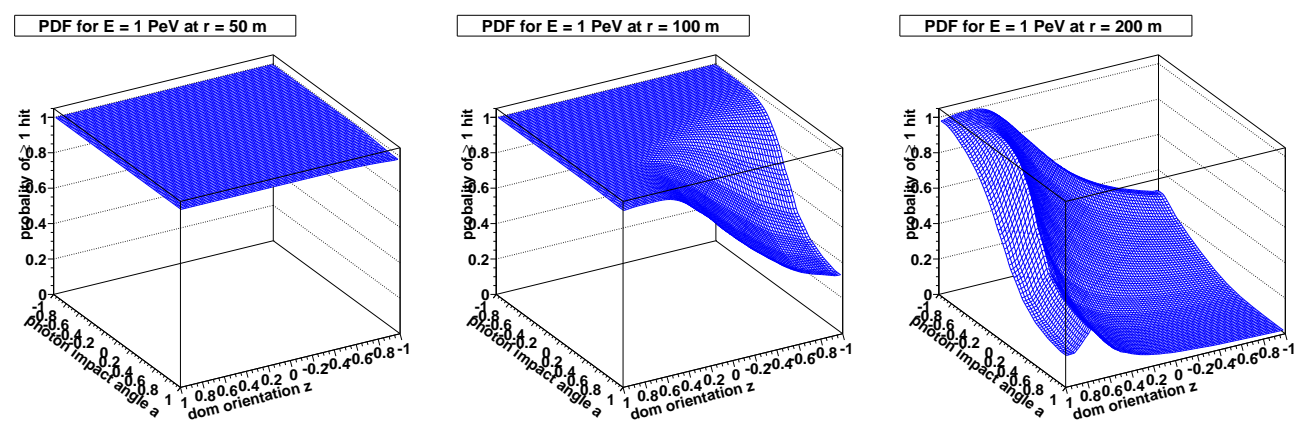

Figure 8: PMT hit probability as function of the photon impact angle and PMT position for different distances to an electro-magnetic shower of $1 \mathrm{PeV}$.

In figure 9, the obtained angular and energy resolutions are shown. All events are reconstructed, of which the badly reconstructed events and misreconstructed atmospheric muon events are rejected using some basic quality cuts using the interaction vertex and basic event information [1]. As can be seen, the shower direction and energy can be reconstructed with an median angular resolution of 1.5 degree and an error of about $5 \%$ in the energy estimate. Despite its simplicity, the presented algorithm already holds very convincing results. Investigations on the use of the timeover-threshold values of the hits rather than only the PMT hit/non hit information show even better results, see [8] for more details. 

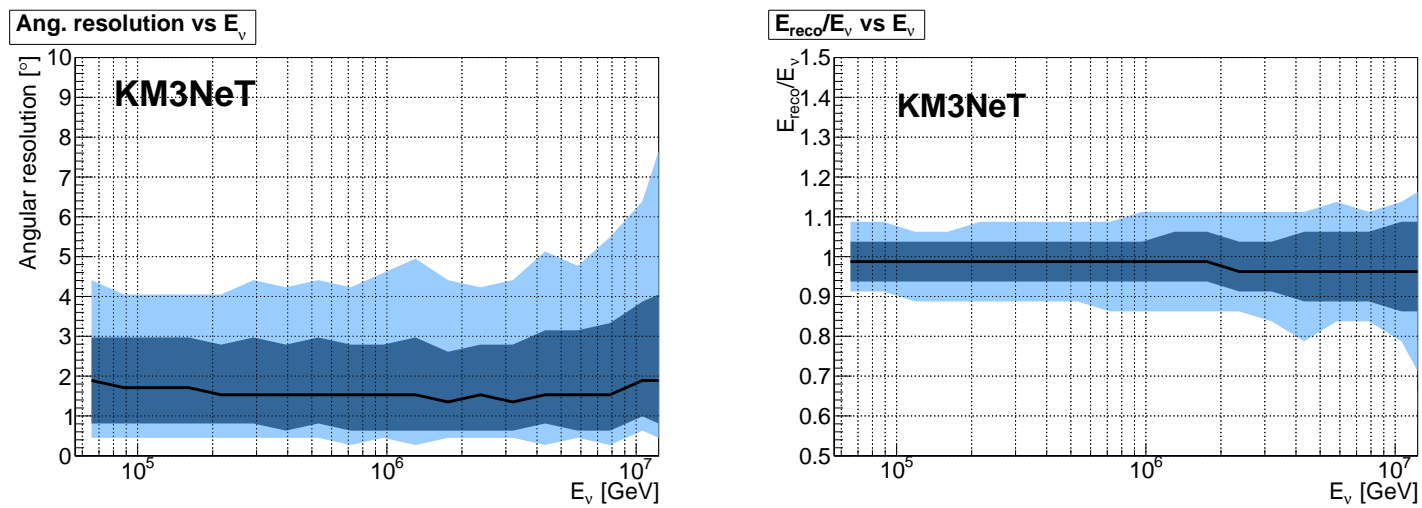

Figure 9: Neutrino-energy dependent median angular (left) and energy (right) errors (solid lines) with $68 \%$ and $90 \%$ quantile bands. The standard deviation of the error in the energy is approximately $5 \%$. The median angular error is about 1.5 degree.

\section{References}

[1] S. Adrían-Martínez et al. (KM3NeT Collaboration), Letter of Intent for ARCA and ORCA, J. Phys. G: Nucl. Part. Phys. 43 (2016), 084001.

[2] A. Heijboer, KM3NeT, Physics and status in these proceedings.

[3] K. Melis, In-Situ Calibration of KM3NeT in these proceedings.

[4] M.Jongen, Measurement of the atmospheric muon flux depth dependence with the first two KM3NeT detection units in these proceedings.

[5] A. Margiotta, Common simulation tools for large volume neutrino detectors, Nucl. Instrum. Meth. 98 (2013), A725.

[6] M. G. Aartsen et al. (IceCube Collaboration), Evidence for high-energy extraterrestrial neutrinos at the IceCube detector. Science 342, 1242856 (2013), [arXiv:1311.5238 [astro-ph.HE]].

[7] F. James, M. Winkler, MINUIT User's Guide: C++ Version (2004)

[8] K. Melis, Reconstruction of High-energy Neutrino-induced Particle Showers in KM3NeT. http://www.scriptiesonline.uba.uva.nl/document/556927 (2014). 